www.jmscr.igmpublication.org

Impact Factor 5.244

Index Copernicus Value: 83.27

ISSN (e)-2347-176x ISSN (p) 2455-0450

crossref DOI: _https://dx.doi.org/10.18535/jmscr/v4i10.104

Journal Of Medical Science And Clinical Research

\title{
Study to Identify the Microorganisms Responsible for Nosocomial Infection in Surgical Intensive Care Unit (SICU) of a Tertiary Care Teaching Hospital of North India
}

\author{
Authors \\ Nowsheen Zaffer ${ }^{1}$, Farooq A.Jan ${ }^{2}$, Haroon Rashid ${ }^{3}$, Mohammed Sarwar Mir ${ }^{4}$, \\ ${ }^{1}$ Senior Resident, ${ }^{2}$ Additional Professor, ${ }^{3}$ Assistant Professor, ${ }^{4}$ Post Graduate \\ Department of Hospital Administration, Sher-i-Kashmir Institute of Medical Sciences, Srinagar, Jammu \& \\ Kashmir, India-190010 \\ Corresponding Author \\ Mohammed Sarwar Mir \\ Email: mohammedsarwarmir@gmail.com
}

\begin{abstract}
Nosocomial infections, also called 'hospital-acquired infections' are infections acquired during hospital care which are not present or incubating at admission. Infections occurring more than 48 hrs after admission are usually considered nosocomial. A prospective study was conducted over a period of one year in the surgical intensive care unit (SICU) of Sher-i-Kashmir Institute of Medical Sciences (SKIMS), Srinagar to identify the organisms responsible for nosocomial infections.

A prospective study was conducted for a period one year in surgical intensive care unit of SKIMS, a tertiary care teaching centre. A total of 190 confirmed cases of nosocomial infection were included in the study. Microbial examination of swab, pus, blood and tracheal aspirate was done to identify the microorganisms responsible for the infections. Acinetobacter baumani was the most common organism identified in swab samples (31\%) and tracheal aspirates (36.9\%) while as $46 \%$ blood cultures were sterile and Klebsella pneumoniae was identified in $13 \%$ of the blood cultures.

Keywords: Nosocomial, Infection, Intensive care unit, Swab, Culture,
\end{abstract}

\section{INTRODUCTION}

Nosocomial infections, also called 'hospitalacquired infections' are infections acquired during hospital care which are not present or incubating at admission. Infections occurring more than 48 hrs after admission are usually considered nosocomial $^{(1)}$. Infections incubating at the time of patients admission to hospital are not nosocomial, they are community acquired ${ }^{(2)}$. Nosocomial infections may involve not only patients but also anyone else who has contact with the hospital including staff members and visitors ${ }^{(3)}$. Infection may present also after patient discharge. In these cases, the patient has become colonized or infected while in hospital, but the pathogen incubation period exceeds the patient's hospital stay. For instance, several studies report that over $50 \%$ of surgical site infections (SSI) manifest post discharge $^{(4)}$.

Intensive care unit is a highly specified and sophisticated area of a hospital which is specifically designed, staffed, located furnished and equipped, dedicated to management of critically sick patient, injuries or complications ${ }^{(5)}$. 
ICU patients are at risk of both acquiring nosocomial infection and for dying ${ }^{(6)}$. Patients in intensive care units (ICUs) are a significant subgroup of all hospitalized patients, accounting for about $25 \%$ of all hospital infections ${ }^{(7)}$.

The prevalence of ICU-acquired infections is significantly higher in developing countries than in industrialized countries, varying between $4.4 \%$ and $88.9 \% .{ }^{(7)}$ Nosocomial infection rates in adult and pediatric ICUs are approx three times higher than elsewhere in hospital. The site of infection and the pathogen involved are directly related to treatment in ICUs ${ }^{(8)}$.

Keeping in mind the impact of nosocomial infection on total costs of care and outcome of care the present study was undertaken at Sher-IKashmir Institute of Medical Sciences [SKIMS], Srinagar to identify the causative micro organisms of nosocomial infections in this hospital. This would lead to rational use of antibiotics and devising appropriate strategies to curb these organisms in the hospital environment.

\section{METHODS}

A prospective study was carried for a period of one year from Oct 2013 to Sep 2014 in the surgical intensive care unit (SICU). All the confirmed cases of nosocomial infection in SICU during the study period were included in this study. The material which was selected for microbiological examination was grouped as under:

- Swab

- Pus

- Tracheal aspirate

- Blood

The data obtained was entered on the proforma and the micro organism responsible viz-a-viz the material used for the sample was enlisted.

\section{RESULTS}

Nosocomial infections (NIs) constitute an important worldwide health problem with high morbidity and mortality rate as well as economic consequences. Nosocomial infections have become especially prominent in intensive care units (ICUs), where the incidence is two to five times greater than in the general inpatient population.

A total of 190 confirmed nosocomial cases where studied

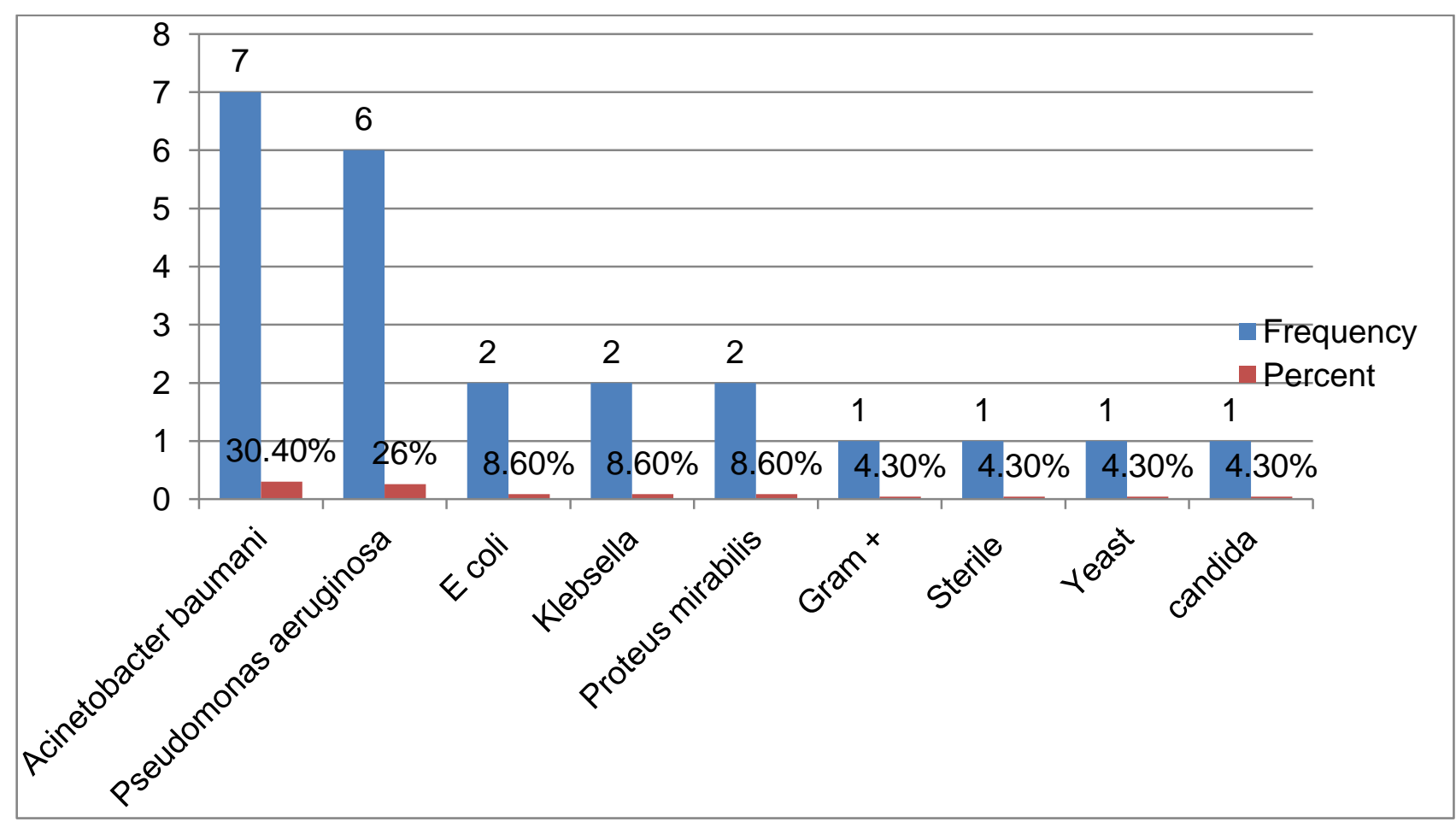

Figure 1: Micro Organisms Found In Swab 
Figure 1 shows In $12.1 \%(\mathrm{n}=23)$ patients who presented with nosocomial infection swab sample was taken for identification of micro organism responsible for nosocomial infection. It revealed that Acineobacter baumani was present in $31 \%$ $(\mathrm{n}=7)$, followed by Pseudomonas aeruginosa in $26 \%(\mathrm{n}=6)$

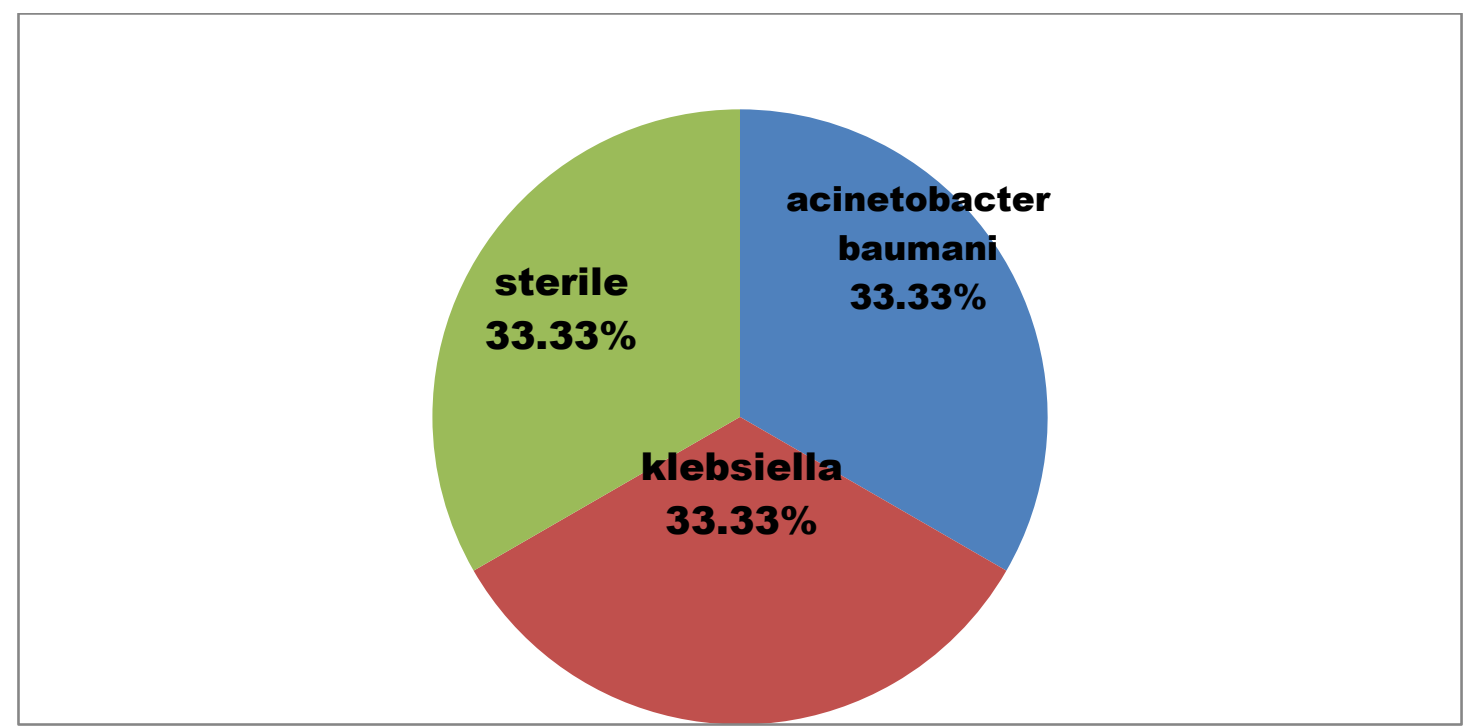

Figure 2: Micro Organisms Found In Pus

Figure 2 -For the identification of micro organism responsible for nosocomial infection in $1.57 \%$ patients $(n=3)$ the pus sample was taken and it showed Acinetobacter baumani and Klebsiella in one each case $(33.33 \% \mathrm{n}=1)$ while it was sterile for one case $(33.33 \% \mathrm{n}=1)$.
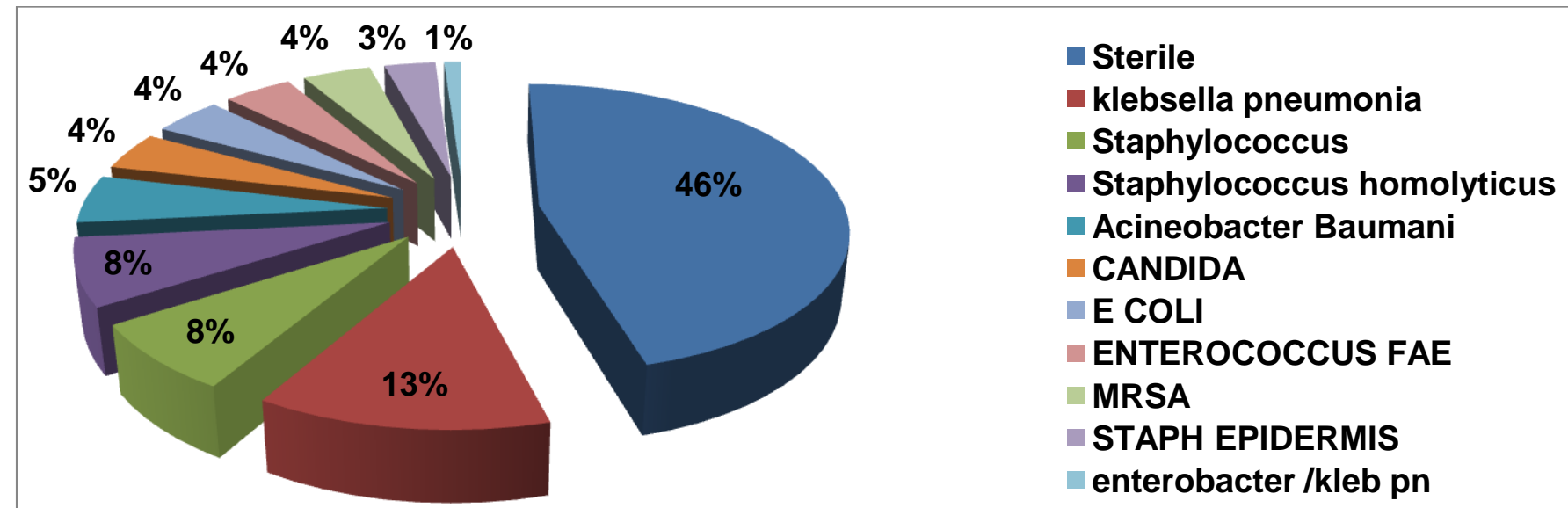

Figure 3: Micro Organisms Found In Blood Culture

Figure 3 shows that Of the total 190 patients diagnosed with nosocomial infection in our study blood culture was done in 94 patients $(49.47 \%)$ $\mathrm{n}=9$, of which 43 samples $(46 \%) \mathrm{n}=43$ proved to be sterile. Klebsiella was seen in 12 patients (13\%) $\mathrm{n}=12$ while staph and staphylococcus hemolyticus infection was found in 7 patients $(8 \%) \mathrm{n}=7$ each. 


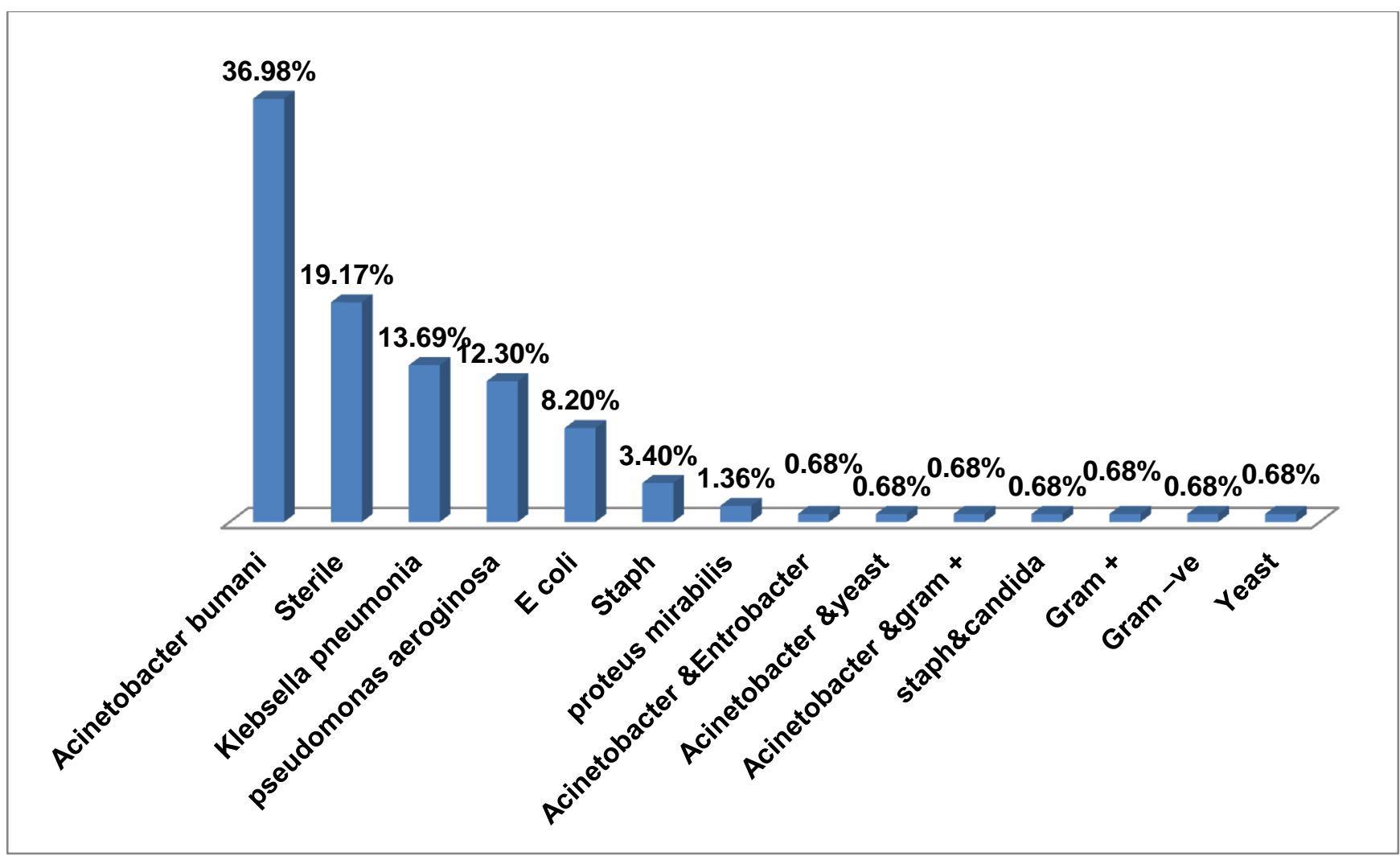

Figure 4: Microorganisms Found In Tracheal Aspirate

Figure 4-In our study tracheal aspirate was taken in 146 patients with nosocomial infection of which $(36.9 \%) \mathrm{n}=54$, patients were affected by Acinetobacter baumani while Klebsiella pneumoniae was found in $13.6 \% \quad(n=20)$, Pseudomonas aeuroginosa was seen in $12.3 \%(\mathrm{n}=$ 18), E coli was seen in $8.2 \%(n=12)$ and $(3.4 \%)$ patients $\mathrm{n}=5$ were affected by Staph and Proteus miribilis was seen in (1.4\%) $\mathrm{n}=2$, one patient $0.68 \%(\mathrm{n}=1)$ each had gram positive, gram negative and yeast infection. (19.17\%) patients $\mathrm{n}=28$ showed sterile culture.

\section{DISCUSSION}

Healthcare-associated infections (HAIs) are a major global problem for patient safety, and are related to significantly increased morbidity, mortality, hospital stays, and costs. The HAIs account for an estimated 1.7 million infections and 99,000 deaths annually in the US. Along with hand hygiene and isolation practices, surveillance is one of the most important steps in controlling HAIs ${ }^{(9)}$.

In $12.1 \% \quad(\mathrm{n}=23)$ patients who presented with nosocomial infection swab sample was taken for identification of micro organism responsible for nosocomial infection. It revealed that Acinetobacter baumani was present in $31 \%(n=7)$, followed by Pseudomonas aeroginosa in $26 \%$ $(n=6)$ and $9 \%(n=2)$ patients each had E.Coli, Klebsella, Proteus mirabilis infection. One patient i.e $4 \%(n=1)$ patient was positive for gram positive bacteria, Candida and yeast while in one case i.e $4 \%(n=1)$ sample was sterile

For the identification of micro organism responsible for nosocomial infection in $1.57 \%$ patients $(n=3)$ the pus sample was taken and it showed Acinetobacter baumani and Klebsiella in one each case $(33.33 \% \mathrm{n}=1)$ while it was sterile for one case $(33.33 \% \quad n=1)$. The soft tissue infection (cellulitis, abscess) was found in 3 patients. Similar findings were revealed by Jan Mohammad et $\mathrm{al}^{(10)}$ in which wound infection was observed in $22(22.7 \%)$ patients referred from different wards, i.e., surgery, gynaecology/ obstetrics and neurosurgery. Organisms detected in such patients were staphylococcus aureus and pseudomonas. The pressure (bed) sores or decubitus ulcers were observed in $27(27.8 \%)$ patients while Suchitra Joyce et al ${ }^{(11)}$ found that 
the commonest etiology for SSI included Staph aureus $\mathrm{n}=45(33 \%)$ of which $14 \% \mathrm{n}=19$ were methicillin resistant staph aureus and enterococcus faecalis (33\%). Pseudomonas Aeruginosa (24.4\% $\mathrm{n}=33)$ E coli $(7.4 \%, \mathrm{n}=10)$ and klebsiellaspp $(1.4 \% \mathrm{n}=2)$ were also isolated.

In majority of studies, gram negative bacteria have been reported as the most common cause of ICU acquired infections. Of the total 190 patients diagnosed with nosocomial infection in our study blood culture was done in 94 patients $(49.47 \%)$ $\mathrm{n}=9$, of which 43 samples $(46 \%) \mathrm{n}=43$ proved to be sterile. Klebsiella was seen in 12 patients (13\%) $\mathrm{n}=12$ while staph and Staphylococcus hemolyticus infection was found in 7 patients (8\%) $n=7$ each. Acinetobacter baumani was found in $(5 \%) \mathrm{n}=5$ and (4\%) $\mathrm{n}=4$ showed Candida, E.Coli, Enterococcus fecalis, methicillin resistant staph respectively. About 3\% $(n=3)$ were positive for staph epidermis and $1 \% \quad(\mathrm{n}=1)$ showed multiple organisms with enterobacter and klebsella, while study byMeliha Merci. ${ }^{(12)}$ revealed following microorganism in blood cultures i.e., Staph aures (33.3\%), enterococcus (20\%), coag negative staph $(13.3 \%)$, candida (13.3\%), klebsella pneumonia (6.7\%) and enterobacter $(6.7 \%)$. Similarly the most common micro organism detected in the study by Jan Mohammad et $\mathrm{al}^{(10)}$ were Coagulase negative Staphylococci [staphylococci epidermis in 1 patient and Staphylococcus saprophyticus in 1 patient], staphylococcus aureus in 9 patients, methicillin resistant staphylococci MRSA in 4 patients, vancomycin resistant enterococci in 4 patients, enterococcus in 2 patients and enterobacter in 01 patient. The source of such bloodstream infections were intravenous cannulae and central venous line. All such pathogens were detected in blood on culture and sensitivity. Similarly study by Oznur AK et $\mathrm{al}^{(13)}$ on ICU infections found 13 patients with staph aueus, 9 with acinetobacter baumani, 8 with Pseudomonas aeruginosa, 5 patients were infected with e coli, 4 patients with Candida and E cloacae respectively while 3 patients had klebsella and enterococcus infection respectively
In our study tracheal aspirate was taken in 146 patients with nosocomial infection of which (36.9\%) $n=54$, patients were affected by Acinetobacter baumani while klebsiella pneumonia was found in $13.6 \%(n=20)$, Pseudomonas aeuroginosa was seen in $12.3 \%(\mathrm{n}=18)$, E coli was seen in $8.2 \%(\mathrm{n}=12)$ and $(3.4 \%)$ patients $\mathrm{n}=5$ were affected by staph and Proteus miribilis was seen in $(1.4 \%) \mathrm{n}=2$, one patient $0.68 \%(\mathrm{n}=1)$ each had gram positive, gram negative and yeast infection. (19.17\%) patients $\mathrm{n}=28$ showed sterile culture. Also there was one case each $(0.68 \%) n=1$ which showed multiple bacteria involved, in these along with Acinetobacter baumani there were enterobacter, yeast and gram positive micro organisms respectively. One case $0.68 \% \quad(n=1)$ was positive for both staph and Candida. Our findings were in line with the study by Ji-Guang Ding_et $\mathrm{al}^{(14)}$ who established that in patients with LRTIs, Acinetobacter baumani and Klebsiella pneumoniae were the most frequently isolated pathogens, followed by Pseudomonas aeruginosa and Staphylococcus aureus, accounting for more than half of the LRTI related pathogen population. Similarly Meliha merci ${ }^{(12)}$ which revealed staph aures in $42.1 \%$, acinetobacter in $28.9 \%$, pseudomonas aeruginosa in $23.6 \%$, klebsella pneumonia in $2.6 \%$ and enterobacter in $2.6 \%$. Although Staphylococcus aureus (S. aureus) was the leading cause of VAP according to the NHSN data, this microorganism was isolated from only $11 \%$ of Emin E et al ${ }^{(15)}$ study of VAP cases. Study by Rasmus Leistner et al ${ }^{(\mathbf{1 6})}$ established that the four most commonly found organisms in cases with LRTI were E.coli(19\%), S.aureus (16\%), Klebsiella spp.(12\%) and P.aeruginosa (7\%). Another study by $\mathrm{J}$ SoléViolán et $\mathrm{al}^{(\mathbf{1 7})}$ revealed that Staphylococcus aureus $(n=26)$, Pseudomonas aeruginosa $(n=25)$ and Haemophilus influenzae $(n=16)$ were the most frequent etiologies for Pneumonia while etiology was polymicrobial in 16 cases (19\%).

\section{SUMMARY}

Healthcare-associated infections (HAIs) are a major global problem for patient safety, and are 
related to significantly increased morbidity, mortality, hospital stays, and costs. Intensive care unit is a highly specified and sophisticated area of a hospital which is specifically designed, staffed, located furnished and equipped, dedicated to management of critically sick patient, injuries or complications. ICU patients are at risk of both acquiring nosocomial infection and for dying. Patients in intensive care units (ICUs) are a significant subgroup of all hospitalized patients, accounting for about $25 \%$ of all hospital infections.

A prospective study was conducted for a period one year in surgical intensive care unit of SKIMS, a tertiary care teaching centre. A total of 190 confirmed cases of nosocomial infection were included in the study. Microbial examination of swab, pus, blood and tracheal aspirate was done to identify the microorganisms responsible for the infections. Acinetobacter baumani was the most common organism identified in swab samples $(31 \%)$ and tracheal aspirates $(36.9 \%)$ while as $46 \%$ blood cultures were sterile and Klebsella pneumonaie was identified in $13 \%$ of the blood cultures.

\section{REFERENCES}

1. Prevention of Hospital acquired infection a practical guide. WHO/CDS/CSR/ EPH. 2002.1.1

2. SirramLata, "nosocomial infection and its control in Hospitals". Control of hospital infections. Jr consultants, India

3. Brachmann PS, "Epidemiology of nosocomial infection". Hospital infection. Edited by John V. Benett and Phillip S. Brachmann4th edition 1998; 1:4.

4. Report on the burden of endemic health care associated infections worldwide. whqlibdoc.who.int/publications/2011/9789 241501507_eng.pdf

5. Patel Kiran B, Patel Disha A, VyasMadhuri S, Patel Ankit Kumar N, Patel Dhaval D, Dhedhi Darshana P, “An evaluation of clinical, supportive and utility services of hospital on quality of patient care in trauma ICU-A six month observational retrospective analysis" Int $\mathbf{J}$ Res Med. 2014; 3(3);144-150

6. Girou $E^{1}$, Stephan F, Novara A, Safar M, Fagon JY, "Risk factors and outcome of nosocomial infections: results of a matched case-control study of ICU patients. ”Am J RespirCrit Care Med. 1998 Apr; 157(4 Pt 1):1151-8.

7. Adamu Sadiq Abubakar, Mohammed Maimadu Barma, Habiba Jimeta Balla, Yusuf SamboTanimu, Goni Baba Waru and Josiah Dibal, "Spectrum of bacterial isolates among intensive care unit patients in a tertiary hospital in northestern Nigeria”.'Ind. J. Sci. Res. and Tech. 2014 2(6):42-47/Abubakar et al

8. Weinstein R A."Nosocomial infection updates special issue". vol 4 no 3 July-sep 1998:416

9. Emin E. Tutuncu, YunusGurbuz, Irfan Sencan, Baris Ozturk, Gonul C. Senturk, Aysegul U. Kilic, "Device-associated infection rates and bacterial resistance in the intensive care units of a Turkish referral hospital".

10. Shaikh JM, Devrajani BR, Shah SZ, Akhund T, Bibi IJ Ayub Med Coll Abbottabad., "Frequency, pattern and etiology of nosocomial infection in intensive care unit: an experience at a tertiary care hospital." 2008 Oct-Dec; 20(4):37-40.J Ayub Med Coll Abbottabad. 2008 Oct-Dec; 20(4):37-40.

11. Suchitra Joyce B. and Lakshmidevi N: "Surgical site infections: Assessing risk factors, outcomes and antimicrobial sensitivity patterns." African Journal of Microbiology Research Vol. 3 (4) pp. 175179 April, 2009. ISSN 1996-0808 @2009 Academic Journals.

12. Meliha Meric, Ayse Willke, Cigdem Caglayan and Kamil Toker: "Intensive care unit-acquired infections: incidence, risk factors and associated mortality in a 
Turkish University Hospital. ’Jpn .J. Infect. Dis., 58,297-302, 2005.

13. OznurAk, AyseBatirel, Serdar Ozer, and Serhan Čolakoğlu, "Nosocomial infections and risk factors in the intensive care unit of a teaching and research hospital: A prospecive cohort study". Med SciMonit. 2011; 17(5): PH29-PH34. Published online 2011 May 1. doi: 10.12659/MSM.881750.

14. Ji-Guang Ding, Qing-Feng Sun, Ke-Cheng Li, Ming-HuaZheng, Xiao-Hui Miao, Wu Ni, Liang Hong, Jin-Xian Yang, Zhan-Wei Ruan, Rui-Wei Zhou, Hai-Jiao Zhou, and Wen-Fei He: "Retrospective analysis of nosocomial infections in the intensive care unit of a tertiary hospital in China during 2003 and 2007. 'BMC Infect Dis. 2009; 9: 115.Published online 2009 Jul 25. doi: 10.1186/1471-2334-9-115.

15. Emin E. Tutuncu, YunusGurbuz, IrfanSencan, BarisOzturk, Gonul C. Senturk, Aysegul U. Kilic, "Deviceassociated infection rates and bacterial resistance in the intensive care units of a Turkish referral hospital".

16. Rasmus Leistner, Linda Kankura, Andy Bloch, DoritSohr, Petra Gastmeier and Christine Geffers : "Attributable costs of ventilator-associated lower respiratory tract infection (LRTI) acquired on intensive care units: a retrospectively matched cohort study"Antimicrobial Resistance and Infection Control 2013, 2:13 . doi:10.1186/2047-2994-2-13.

17. J SoléViolán, C Sánchez-Ramírez, A PadrónMújica, JA CardeñosaCendrero, J Arroyo Fernández and F Rodríguez de Castro, "Impact of nosocomial pneumonia on the outcome of mechanically-ventilated patients".Crit Care. 1998; 2(1): 19-23. Published online 1998 Mar 12. doi: $10.1186 /$ cc119. 